\title{
Impact of loganin on pro-inflammatory cytokines and depression- and anxiety-like behaviors in male diabetic rats
}

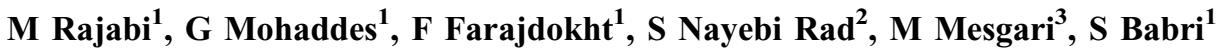 \\ ${ }^{1}$ Neuroscience Research Center (NSRC), Tabriz University of Medical Sciences, Tabriz, Iran \\ ${ }^{2}$ Faculty of Medicine, Tehran University of Medical Sciences, Tehran, Iran \\ ${ }^{3}$ Drug Applied Research Center, Tabriz University of Medical Sciences, Tabriz, Iran
}

Received: June 3, 2017

Accepted: January 9, 2018

\begin{abstract}
Behavioral disturbances are observed in most patients suffering from diabetes. According to some evidence, pro-inflammatory cytokines have a key role both in diabetes and behavioral disorders, such as anxiety and depression. In this study, the effect of chronic administration of loganin, as a bioflavonoid, was investigated on pro-inflammatory cytokines and depression- and anxiety-like behaviors in streptozotocin-induced diabetes in male Wistar rats. Blood levels of interleukin-6 (IL-6) and tumor necrosis factor-alpha (TNF- $\alpha$ ) were assessed by enzymelinked immunosorbent assay method. Depression- and anxiety-like behaviors were evaluated by forced swimming test (FST), elevated plus maze (EPM), and open field test (OFT), respectively. Body weight was also measured before the interventions and after the experiments in all groups. Our findings show that loganin-treated animals had significantly lower serum concentrations of IL-6 and TNF- $\alpha$ compared with the diabetic group. In the EPM test, loganin treatment significantly increased the percentage of the open arm time and open arm entries. Moreover, loganin treatment significantly decreased the grooming time and restored distance traveled and center crossing in the OFT. However, it decreased immobility time in the FST. Loganin treatment also significantly restored body weight gain and attenuated blood glucose changes in the diabetic rats. These results indicate that loganin possibly alleviates depression- and anxiety-like behaviors associated with diabetes through lowering the blood glucose and pro-inflammatory cytokine levels. More research is required to show the exact mechanism of antidepressant and anxiolytic effects of loganin in diabetes.
\end{abstract}

Keywords: diabetes, anxiety, depression, loganin, IL-6, TNF- $\alpha$

\section{Introduction}

Most people suffering from diabetes have a dysfunction in their nervous system (23). Recent researches focus on the relationship between diabetes and behavioral changes $(8,42)$. Depression and anxiety are two common psychological disorders in people with diabetes (5). Anxiety is one of the most common psychiatric disorders. It affects many aspects of life and is highly comorbid with depression (47). Patients with anxiety exhibit significant risk for load inability, which can also be seen in chronic diseases, like diabetes mellitus $(38,41)$. In recent years, some of the studies have shown that the prevalence of symptoms of anxiety and generalized anxiety disorders has increased in patients with diabetes $(9,41)$. There is also

\footnotetext{
Corresponding author: Prof. Dr. Shirin Babri, $\mathrm{PhD}$

Neuroscience Research Center (NSRC), Tabriz University of Medical Sciences

Golgasht Street, Azadi Avenue, Tabriz 5166614756, Iran

Phone/Fax: +98 413336 4664; E-mail: shirinb46@yahoo.com
} 
evidence that patients with type 2 diabetes display depression occurrence twice as high as the general population (40).

To describe the pathogenesis of depression, several mechanisms have been suggested including abnormalities in the circadian rhythm, increased inflammatory cytokines, hypercortisolemia, and deficiency of monoamines. It has also been suggested that oxidative stress is increased in patients with depression $(4,28)$. Moreover, many studies have shown that dysregulation of the immune system is associated with depression and anxiety disorders $(15,20)$. Therefore, according to the evidence, there is a close relationship between diabetes and depression and anxiety disorders. In addition, previous studies have suggested an association between impaired glucose control and levels of pro-inflammatory mediators $(30,46)$.

Dysregulation in both the acquired and the innate immune systems is coupled to the onset of insulin resistance and type 2 diabetes $(10,43)$. Poorly controlled diabetes leads to hyperglycemia that affects the immune system and increases pro-inflammatory mediators, such as tumor necrosis factor-alpha (TNF- $\alpha$ ), interleukin-6 (IL-6), and C-reactive protein $(6,34,44)$. Therefore, pro-inflammatory factors could be the common underlying risk factors for both diabetes and behavioral disorders.

Loganin is one of the various components of Cornus officinalis, which is highly respected in Chinese medicine. Previous studies have shown that loganin has antihyperglycemic (39), anti-inflammatory, anti-shock, and anti-arrhythmic effects $(48,50)$. Our previous research also showed that loganin significantly decreased oxidative stress products in the central nervous system of diabetic rats (1). Given the fact that loganin possesses neuroprotective properties and cognitive enhancing effects and is capable of regulating immune system function, there are suggestions about the therapeutic use of loganin in the treatment of neurodegenerative diseases $(1,25)$.

The aim of this study was to investigate the effect of chronic administration of loganin on the immune system and depression- and anxiety-like behaviors in the diabetic rats.

\section{Materials and Methods}

\section{Rats}

Adult male Wistar rats $(N=40)$, weighing 200-250 g, were obtained from Animal House of Tabriz, University of Medical Sciences. The rats were housed three per cage in a standard condition with 12-h light/dark cycle, $20-22{ }^{\circ} \mathrm{C}, 45 \%-55 \%$ humidity, free access to food and water, and lights were switched on at 6:00 a.m. (2). All procedures were carried out in accordance with the National Institutes of Health Guide for the Care and Use of Laboratory Animals. All experimental procedures were also approved by Regional Ethics Committee of Tabriz University of Medical Sciences.

\section{Experimental design}

Seven days after adaptation, rats were randomly divided into five groups $(n=8)$ : control (con), diabetic plus saline (dia + saline), diabetic plus imipramine (dia + imipr), diabetic plus diazepam (dia + diaz), and diabetic plus loganin $($ dia $+\log )$. Experimental diabetes was induced by intraperitoneal (i.p.) injection of a single dose of $60 \mathrm{mg} / \mathrm{kg}$ streptozotocin (STZ) (Tocris, UK) (35). Fasting blood glucose levels were determined $72 \mathrm{~h}$ after STZ injection, using a glucometer (Roche, Germany). Blood samples were collected from the tail vein of 
overnight $(10 \mathrm{~h})$ fasted rats. Rats were considered diabetic, if blood glucose levels exceeded $300 \mathrm{mg} / \mathrm{dl}$. Confirming diabetes (on day 4 following STZ injection), rats received saline (1 ml), imipramine (15 mg/kg i.p.) (ScienceLab, USA) (7), or diazepam (1 mg/kg i.p.) (Sigma, USA), $30 \mathrm{~min}$ before behavioral tests (14). The rats in the dia $+\log$ group received $40 \mathrm{mg} / \mathrm{kg}$ of loganin per os (Extrasynthese, France), which was administered at 9:00 a.m. for 10 consecutive days. On day 10, the behavioral tests were performed an hour after the loganin administration.

\section{Behavioral tests}

Forced swimming test (FST). Behavioral tests were performed in the morning (10 a.m.-12 p.m.) under low illumination. Briefly, rats were individually placed in a Plexiglas cylinder $(46 \mathrm{~cm}$ in height with a $21-\mathrm{cm}$ internal diameter) filled with water $\left(23 \pm 1^{\circ} \mathrm{C}\right)$ to a depth of $30 \mathrm{~cm}$. Water was changed between each swim session. In the pretest session, rats were placed in the water for $15 \mathrm{~min}$ (first exposure). Then, $24 \mathrm{~h}$ later, a test session was performed and immobility behavior of rats was recorded for $5 \mathrm{~min}$ (24). Immobility was defined as the ceasing of struggling and remaining floating motionless in the water by making movements only necessary to keep the rat's head above the water. Decreased immobility time is an indicator of an antidepressant-like effect. A blinded experimenter to the treatments analyzed video recording.

Elevated plus maze (EPM). The EPM test, one of the well-known methods for testing of anxiety in rodents, was performed as previously described by our laboratory. Briefly, EPM was a cross-shaped maze whose apparatus consisted of two open arms $(50 \mathrm{~cm} \times 10 \mathrm{~cm})$ and two enclosed arms $(50 \mathrm{~cm} \times 10 \mathrm{~cm} \times 40 \mathrm{~cm})$. The maze was elevated to a height of $50 \mathrm{~cm}$. The rats were individually placed in the center of the maze facing an open arm and allowed 5 min of free exploration. All sessions were videotaped and the number of entries and the time spent in the open and enclosed arms were measured. Entry was defined when all four paws of rats are set in the arms. Percentage of entries into open arms and time spent in open arms were calculated as the standard anxiety indices as follows: (i) percentage of the open arm entries (\% OAE) [(the ratio of entries into the open arms to total entries) $\times 100$ ] and (ii) percentage of the open arm (\% OAT) [(the ratio of time spent in the open arms to total time spent in any arms $) \times 100]$. Total arm entries were also considered as the index of locomotor activity $(11,24)$.

Open field test (OFT). The OFT is often used to assess anxiety-like behaviors in rodents. The open field apparatus was an arena $(50 \times 50 \times 40 \mathrm{~cm})$ with black plywood walls and a brown floor divided into nine squares by black lines. Each rat was gently placed in the center of the arena and allowed to freely explore the novel environment for $5 \mathrm{~min}$. All behaviors including distance traveled $(\mathrm{cm})$, time spent in the center (s), grooming time (s), and the number of fecal boli were recorded during the test period by an observer blinded to the treatments. Between each session, the arena was cleaned with $70 \%$ ethanol and dried (32).

Blood sampling. At the end of the experiments, all the rats were sacrificed between 10:00 a.m. and 12:00 p.m. Blood samples were collected from the heart apex and centrifuged at $4{ }^{\circ} \mathrm{C}$ at 4,000 rpm. Serum levels of IL- 6 and TNF- $\alpha$ were measured using enzyme-linked immunosorbent assay kits (eBioscience, San Diego, CA) according to the manufacturer's protocols.

\section{Statistical analysis}

Results are presented as mean \pm standard error of the mean. All data were statistically analyzed by the SPSS16 software package. For body weight changes, paired sample $t$-test, 
and for serum levels of IL-6, Kruskal-Wallis followed by Mann-Whitney's post-hoc test was used. For all other variables, one-way analysis of variance followed by post-hoc Tukey's test was used for multiple comparisons. Statistical significance was regarded as $p<0.05$.

\section{Results}

Loganin improved weight gain and blood glucose levels in diabetic rats

Body weight was measured before the interventions and after the experiments in all rats. There was no statistically significant difference among groups in body weight before the experiments (Fig. 1A). We found that the rate of body weight in the dia + saline group was significantly $(p<0.01)$ lower than their body weight before the interventions. The administration of loganin significantly $(p<0.05)$ improved body weight gain in the dia $+\log$ group.

Our results also demonstrated that blood glucose increased in the diabetic group $(p<0.01)$ (Fig. 1B). However, loganin treatment significantly $(p<.05)$ decreased fasting blood glucose in the dia $+\log$ group in comparison with the dia + saline group.

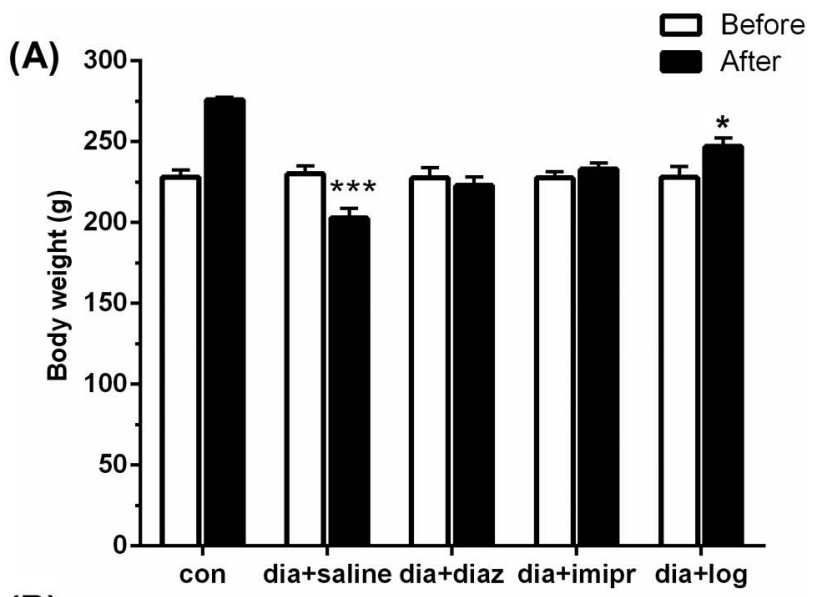

(B)

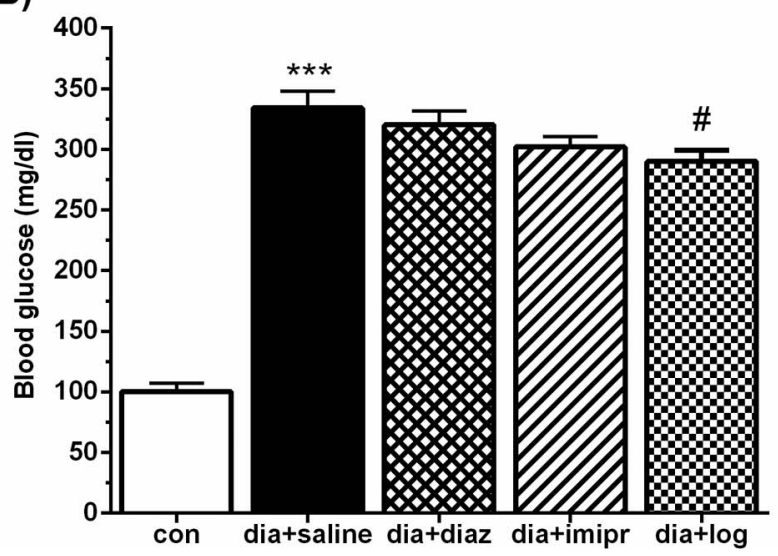

Fig. 1. Effect of loganin on (A) body weight; paired $t$-test: ${ }^{*} p<0.05$, $* * * p<0.001$, and (B) blood glucose levels in different groups. Data are expressed as mean \pm SEM $(n=8)$. con: control; dia + saline: diabetic plus saline; dia + imipr: diabetic plus imipramine; dia + diaz: diabetic plus diazepam $1 \mathrm{mg} / \mathrm{kg}$; dia + log: diabetic plus loganin. $* * * p<0.001$ versus control group and ${ }^{\#} p<0.05$ versus dia + saline group 


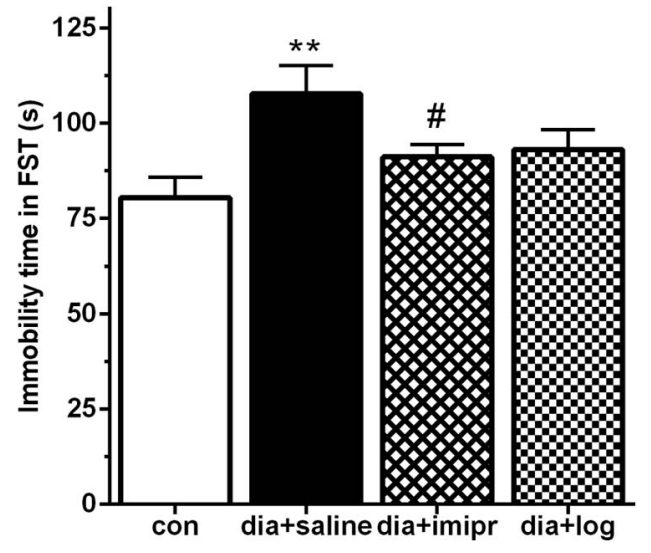

Fig. 2. Effect of loganin on immobility time between different groups in FST. Data are expressed as mean $\pm \operatorname{SEM}(n=8)$. con: control;

dia + saline: diabetic plus saline; dia + imipr: diabetic plus imipramine; dia + diaz: diabetic plus diazepam $1 \mathrm{mg} / \mathrm{kg}$; dia + log: diabetic plus loganin. ${ }^{* *} p<0.01$ versus control, ${ }^{\#} p<0.05$ versus dia + saline group

Loganin decreased depression-like behaviors in diabetic rats

According to the results, the immobility time in the dia + saline group was significantly $(p<0.01)$ more than in the con group (Fig. 2). There was also a significant $(p<0.05)$ difference in immobility time between dia + imipr and dia + saline groups (Fig. 2). Moreover, immobility time in the dia $+\log (p=0.06)$ group was less than that in the dia + saline group, although it was not significant.

Loganin decreased anxiety-like behaviors in EPM and OFT in diabetic rats EPM. Statistically significant differences were observed in the \% OAT $(p<0.01$, Fig. $3 \mathrm{~A})$ and $\%$ OAE $(p<0.05$, Fig. 3B) in the dia + saline group compared with the con group. However, loganin $(40 \mathrm{mg} / \mathrm{kg})$ administration significantly $(p<0.05)$ increased \% OAT and $\%$ OAE in the dia + loganin group when compared with the dia + saline rats. Nevertheless, there was no significant change in \% OAT and \% OAE between the dia + diaz and dia + saline groups. Moreover, there were no significant differences in locomotor activity among four groups (Fig. 3C). These results showed that diabetic rats exhibited an increased amount of anxiety-like behaviors, which was alleviated by loganin.

OFT. Our results showed that diabetic rats had significantly $(p<0.01)$ less center-crossing time (Fig. 4A), lower distance traveled (Fig. 4B), and more grooming time (Fig. 4C) and fecal boli number (Fig. 4D) than the con animals. Importantly, loganin treatment significantly $(p<0.05)$ restored center crossing, distance traveled, and decreased the grooming time compared with the diabetic group. Similarly, diazepam treatment significantly $(p<0.05)$ increased center-crossing time and distance traveled, and decreased fecal boli number in comparison with the diabetic group.

\section{Loganin decreased serum concentration of IL-6 and TNF- $\alpha$ in diabetic rats}

This study showed that induction of diabetes significantly $(p<0.01)$ increased the serum concentrations of IL-6 (Fig. 5A) and TNF- $\alpha$ (Fig. 5B) when compared with the con group. Nevertheless, loganin treatment significantly $(p<0.05)$ reduced serum levels of IL-6 (Fig. 5A) and TNF- $\alpha$ (Fig. 5B) in diabetic rats. Similarly, administration of imipramine to diabetic rats significantly reduced serum levels of IL-6 $(p<0.01$, Fig. 5A) and TNF- $\alpha$ $(p<0.05$, Fig. 5B). 

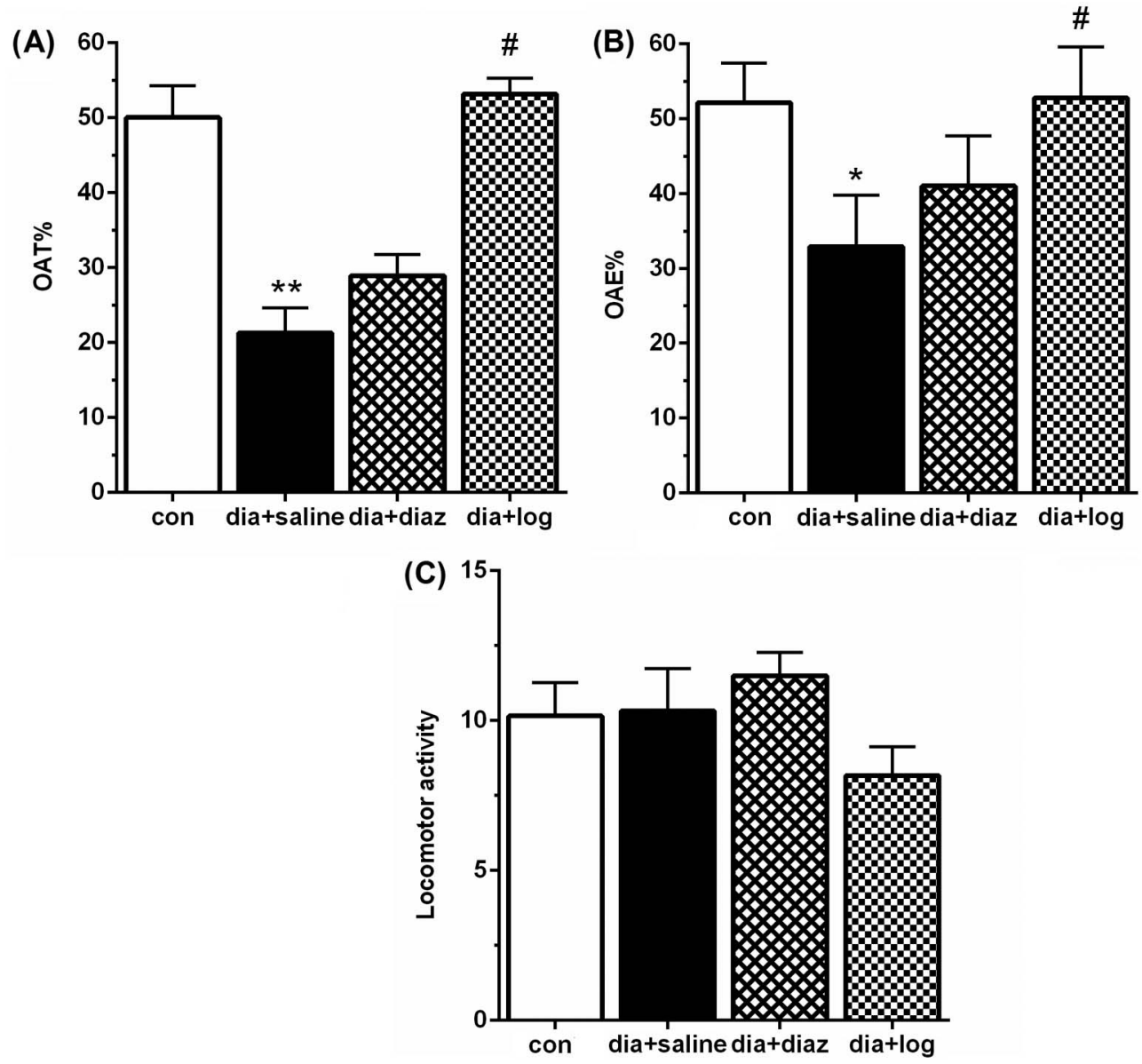

Fig. 3. Effect of loganin on the percentage of open arm time (\% OAT) (A), the percentage of open arm entries $(\% \mathrm{OAE})(\mathrm{B})$, and locomotor activity (C) between different groups in EPM. Data are expressed as mean $\pm \mathrm{SEM}$ $(n=8)$. con: control; dia + saline: diabetic plus saline; dia + imipr: diabetic plus imipramine; dia + diaz: diabetic plus diazepam $1 \mathrm{mg} / \mathrm{kg}$; dia $+\log$ : diabetic plus loganin. ${ }^{*} p<0.05$ and ${ }^{*} p<0.01$ versus control, ${ }^{\#} p<0.05$ versus dia + saline group

\section{Discussion}

Anxiety and depression are common comorbid conditions in type 1 (18) and type 2 (5) diabetes. Although the relationship between type 1 diabetes and anxiety disorders has been demonstrated earlier (18), the nature of this relationship is yet to be elucidated (33). Previous studies have suggested that fear of future hypoglycemic episodes results in high levels of anxiety and stress in the patients suffering from diabetes $(17,21)$.

There is also an interrelationship between the severity of diabetes and depressive mood. Poor glucose control and diabetes complications result in an increased prevalence of depression in patients (45). Inflammation is a mechanistic link between depression and diabetes (43). In this study, we investigated the effect of loganin on the serum levels of IL-6 
(A)

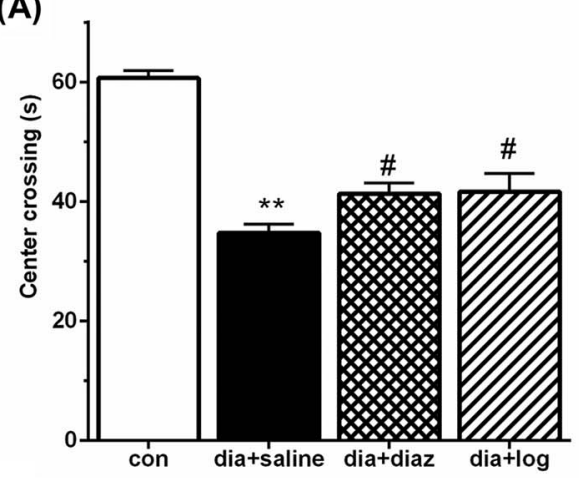

(C)

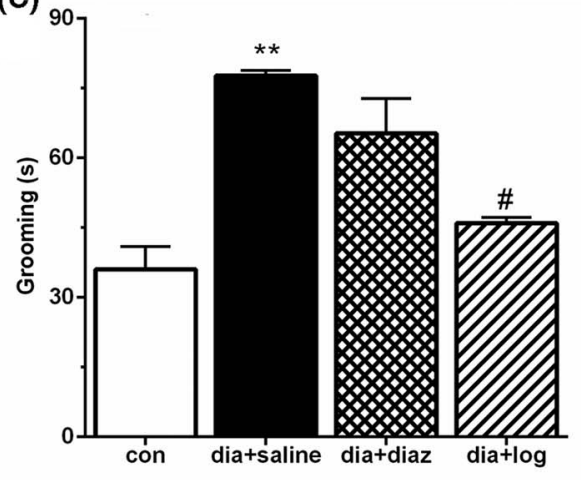

(B)

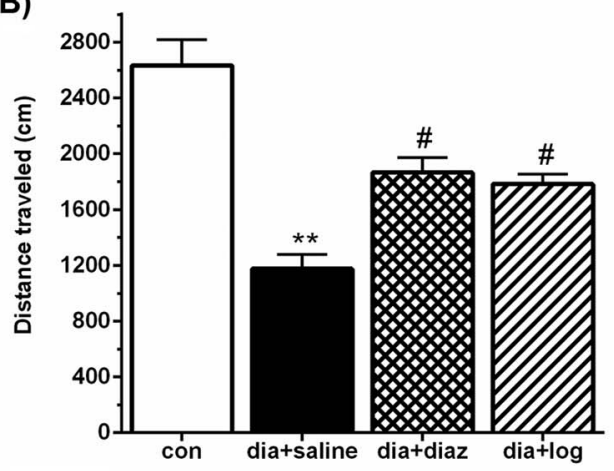

(D)

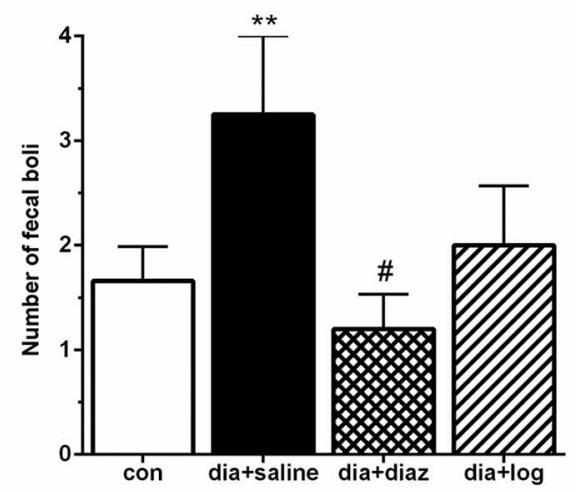

Fig. 4. Effect of loganin on distance traveled (A), center crossing (B), grooming (C), and number of fecal boli (D) among different groups in OFT. Data are expressed as mean $\pm \operatorname{SEM}(n=8)$. con: control; dia + saline: diabetic plus saline; dia + imipr: diabetic plus imipramine; dia + diaz: diabetic plus diazepam $1 \mathrm{mg} / \mathrm{kg}$; dia + log: diabetic plus loganin. ${ }^{*} p<0.05$ and ${ }^{*} p p<0.01$ versus control, ${ }^{\#} p<0.05$ versus dia + saline group

and TNF- $\alpha$, as pro-inflammatory cytokines, and depression- and anxiety-like behaviors in STZ-induced type 1 diabetes.

This study showed that treatment with loganin $(40 \mathrm{mg} / \mathrm{kg})$ for 10 days increased the body weight and reduced blood glucose levels in diabetic rats. Similarly, Liu et al. (27) showed in their study that loganin treatment improved body weight gain and reduced blood glucose levels in diabetic mice. In diabetes, chronic hyperglycemia leads to oxidative stress by increasing reactive oxygen species (ROS) production and glucose autooxidation (13). High levels of ROS trigger protein-kinase $\mathrm{C}$ production through diacylglycerol and elevate production of cytokines involved in inflammation, specifically IL-6, which is known to be the essential intermediary of acute inflammatory response (37). Our previous study demonstrated that loganin decreased oxidative stress in diabetic rats (1).

The results of FST indicated that diabetes increased immobility time, as an index of depression-like behaviors. These results are in line with a previous study reporting that diabetic rats show depression-like behaviors in FSTs (16). Imipramine is one of the most effective drugs for the treatment of depression and is used as a positive control in studies on depression (7). In this study, imipramine and loganin ( $40 \mathrm{mg} / \mathrm{kg} / 10$ days) reduced immobility 


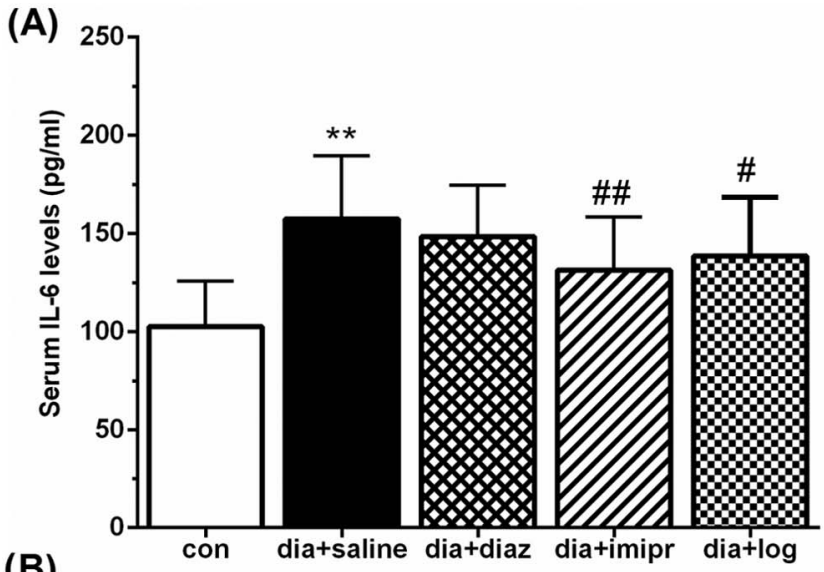

(B)

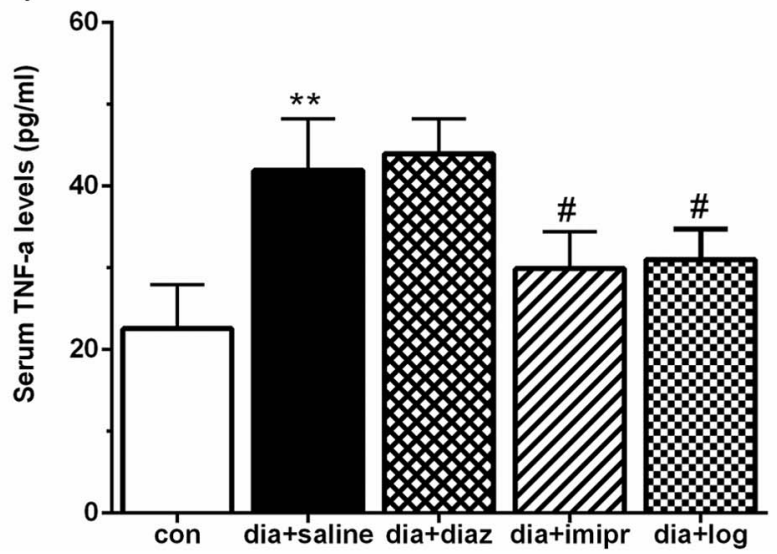

Fig. 5. Effect of loganin on serum concentrations of IL-6 (A) and TNF- $\alpha$ (B) in different groups. Data are expressed as mean \pm SEM $(n=8)$. con: control; dia + saline: diabetic plus saline; dia + imipr: diabetic plus imipramine; dia + diaz: diabetic plus diazepam $1 \mathrm{mg} / \mathrm{kg}$; dia + log: diabetic plus loganin. ${ }^{*} p<0.01$ versus control, ${ }^{\#} p<0.05,{ }^{\# \#} p<0.01$ versus $\mathrm{dia}+$ saline group

time in FST, although there was no significant difference between dia + loganin and dia + saline groups, possibly due to an inadequate dose of loganin for treatment.

Anxiety behaviors are one of the most common psychological disorders. Smith et al. (41) indicated that diabetes is associated with anxiety behaviors. EPM and OFT are reliable tests for investigation of anxiety-like behaviors in rodents (32). In this study, EPM results showed that rats in the diabetic groups exhibited more entries into the closed arms and time spent in the closed arms, which were reversed by loganin treatment. Frassetto et al. (14) used diazepam $(1 \mathrm{mg} / \mathrm{kg})$ as a positive control in their study for its anxiolytic effect and showed that administration of diazepam increased the percentage of entries and time spent in the open arms when compared with diabetic rats. This study has failed to demonstrate this result and there was no apparent anxiolytic activity of diazepam in diabetic rats in EPM. Ramanathan et al. (36) also demonstrated that diazepam $(1 \mathrm{mg} / \mathrm{kg})$ does not have an anxiolytic activity in diabetic rats. Moreover, OFT results in the diabetic rats showed less center-crossing time, lower distance traveled, and more grooming time and defecation. These results indicated that diabetes increased anxiety-like behaviors. In contrast to the EPM results, the results of OFT showed that diazepam significantly increased center-crossing time, distance traveled, and decreased defecation. On the other hand, administration of loganin markedly attenuated these changes in the OFT. 
Increased anxiety-like behaviors in diabetic rats were associated with higher levels of blood glucose, IL-6, and TNF- $\alpha$. A previous study also specified that administration of TNF- $\alpha$ to an animal-induced insulin resistance, whereas TNF- $\alpha$ neutralization improved insulin sensitivity (31). It has been shown that activation of the inflammatory system is related to the behavioral disorders. Inflammation and oxidative stress have major roles in the pathogenesis of depression (12), anxiety (47), and diabetes (49). The results of meta-analysis studies showed that pro-inflammatory cytokines, such as IL- 6 and TNF- $\alpha$, have an important role in the molecular mechanism of depression- and anxiety-like behaviors $(19,29)$. In this study, depression- and anxiety-like behaviors were accompanied by higher serum levels of IL- 6 and TNF- $\alpha$ in the diabetic rats. However, loganin treatment decreased serum IL- 6 and TNF- $\alpha$ concentrations and improved depression- and anxiety-like behaviors. In agreement with the previous studies $(3,22,26)$, our findings also showed that loganin has an anti-inflammatory effect.

Based on the above documents, loganin treatment decreased depression- and anxietylike behaviors in the diabetic rats, possibly through attenuation of blood glucose and pro-inflammatory cytokine levels. Further investigations are required to determine the exact physiological mechanisms leading to antidepressant and anxiolytic effects of loganin. To our knowledge, this was the first study performed to clarify the effects of loganin in depressionand anxiety-like behaviors in the diabetic rats.

\section{Acknowledgements}

This study was financially supported by the Neuroscience Research Center (NSRC) of Tabriz University of Medical Sciences.

\section{REFERENCES}

1. Babri S, Azami SH, Mohaddes G: Effect of acute administration of loganin on spatial memory in diabetic male rats. Adv. Pharm. Bull. 3, 91-95 (2013)

2. Babri S, Doosti MH, Salari AA: Strain-dependent effects of prenatal maternal immune activation on anxiety- and depression-like behaviors in offspring. Brain Behav. Immun. 37, 164-176 (2014)

3. Carrillo-Ocampo D, Bazaldúa-Gómez S, Bonilla-Barbosa JR, Aburto-Amar R, Rodríguez-López V: Anti-inflammatory activity of iridoids and verbascoside isolated from Castilleja tenuiflora. Molecules 18, 12109-12118 (2013)

4. Chung CP, Schmidt D, Stein CM, Morrow JD, Salomon RM: Increased oxidative stress in patients with depression and its relationship to treatment. Psychiatry Res. 206, 213-216 (2013)

5. Collins M, Corcoran P, Perry I: Anxiety and depression symptoms in patients with diabetes. Diabet. Med. 26, 153-161 (2009)

6. Dandona P, Aljada A, Bandyopadhyay A: Inflammation: the link between insulin resistance, obesity and diabetes. Trends Immunol. 25, 4-7 (2004)

7. Dolati K, Rakhshandeh H, Shafei MN: Evaluation of antidepressant effect of ethanolic extract of Rosa damascena using forced swimming test. Avicenna J. Phytomed. 2, 46-51 (2011)

8. Edwards JL, Vincent AM, Cheng HT, Feldman EL: Diabetic neuropathy: mechanisms to management. Pharmacol. Ther. 120, 1-34 (2008)

9. Engum A: The role of depression and anxiety in onset of diabetes in a large population-based study. Psychosom. Res. 62, 31-38 (2007)

10. Eyre HA, Air T, Pradhan A, Johnston J, Lavretsky H, Stuart MJ, Baune BT: A meta-analysis of chemokines in major depression. Prog. Neuropsychopharmacol. Biol. Psychiatry 68, 1-8 (2016)

11. Farajdokht F, Babri S, Karimi P, Alipour MR, Bughchechi R, Mohaddes G: Chronic ghrelin treatment reduced photophobia and anxiety-like behaviors in nitroglycerin-induced migraine: role of pituitary adenylate cyclaseactivating polypeptide. Eur. J. Neurosci. 45, 763-772 (2017) 
12. Farajdokht F, Soleimani M, Mehrpouya S, Barati M, Nahavandi A: The role of hepcidin in chronic mild stressinduced depression. Neurosci. Lett. 588, 120-124 (2015)

13. Francés DE, Ingaramo PI, Ronco MT, Carnovale CE: Diabetes, an inflammatory process: oxidative stress and TNF-alpha involved in hepatic complication. J. Biomed. Sci. Eng. 6, 33682 (2013)

14. Frassetto SS, Alves IO, Santos MM, Schmidt AE, Lopes JJ, Oliveira PA, Vinagre AS, Pereira P: Absence of sibutramine effect on spontaneous anxiety in rats. Arq. Bras. Endocrinol. Metab. 54, 375-380 (2010)

15. Glaser R, Kiecolt-Glaser JK: Stress-induced immune dysfunction: implications for health. Nat. Rev. Immunol. 5, 243-251 (2005)

16. Gomez R, Huber J, Tombini G, Barros H: Acute effect of different antidepressants on glycemia in diabetic and non-diabetic rats. Braz. J. Med. Biol. Res. 34, 57-64 (2001)

17. Gonder-Frederick LA, Fisher CD, Ritterband LM, Cox DJ, Hou L, Dasgupta AA, Clarke WL: Predictors of fear of hypoglycemia in adolescents with type 1 diabetes and their parents. Pediatr. Diabetes 7, 215-222 (2006)

18. Herzer M, Hood KK: Anxiety symptoms in adolescents with type 1 diabetes: association with blood glucose monitoring and glycemic control. J. Pediatr. Psychol. 35, 415-425 (2010)

19. Howren MB, Lamkin DM, Suls J: Associations of depression with C-reactive protein, IL-1, and IL-6: a metaanalysis. Psychosom. Med. 71, 171-186 (2009)

20. Irwin MR, Miller AH: Depressive disorders and immunity: 20 years of progress and discovery. Brain Behav. Immun. 21, 374-383 (2007)

21. Kamps JL, Roberts MC, Varela RE: Development of a new fear of hypoglycemia scale: preliminary results. J. Pediatr. Psychol. 30, 287-291 (2005)

22. Kim M-J, Bae G-S, Jo I-J, Choi S-B, Kim D-G, Shin J-Y, Lee S-K, Kim M-J, Shin S, Song H-J: Loganin protects against pancreatitis by inhibiting NF- $\mathrm{BB}$ activation. Eur. J. Pharmacol. 765, 541-550 (2015)

23. Kodl CT, Seaquist ER: Cognitive dysfunction and diabetes mellitus. Endocr. Rev. 29, 494-511 (2008)

24. Kosari-Nasab M, Babri S, Fatehi-Gharehlar L, Doosti M-H, Pakzad S: Involvement of GABAergic system in regulation of the anxiolytic- and antidepressant-like effects of Scrophularia striata extract in rats. Pharm. Biol. 51, 581-588 (2013)

25. Lee KY, Sung SH, Kim SH, Jang YP, Oh TH, Kim YC: Cognitive-enhancing activity of loganin isolated from Cornus officinalis in scopolamine-induced amnesic mice. Arch. Pharm. Res. 32, 677-683 (2009)

26. Li M, Shang X, Zhang R, Jia Z, Fan P, Ying Q, Wei L: Antinociceptive and anti-inflammatory activities of iridoid glycosides extract of Lamiophlomis rotata (Benth.) Kudo. Fitoterapia 81, 167-172 (2010)

27. Liu K, Xu H, Lv G, Liu B, Lee MKK, Lu C, Lv X, Wu Y: Loganin attenuates diabetic nephropathy in C57BL/6J mice with diabetes induced by streptozotocin and fed with diets containing high level of advanced glycation end products. Life Sci. 123, 78-85 (2015)

28. Lopresti AL, Maker GL, Hood SD, Drummond PD: A review of peripheral biomarkers in major depression: the potential of inflammatory and oxidative stress biomarkers. Prog. Neuropsychopharmacol. Biol. Psychiatry 48, 102-111 (2014)

29. Maes M, Song C, Lin A, De Jongh R, Van Gastel A, Kenis G, Bosmans E, De Meester I, Benoy I, Neels H: The effects of psychological stress on humans: increased production of pro-inflammatory cytokines and Th1-like response in stress-induced anxiety. Cytokine 10, 313-318 (1998)

30. Mirza S, Hossain M, Mathews C, Martinez P, Pino P, Gay JL, Rentfro A, Mccormick JB, Fisher-Hoch SP: Type 2-diabetes is associated with elevated levels of TNF-alpha, IL-6 and adiponectin and low levels of leptin in a population of Mexican Americans: a cross-sectional study. Cytokine 57, 136-142 (2012)

31. Moller DE: Potential role of TNF- $\alpha$ in the pathogenesis of insulin resistance and type 2 diabetes. Trends Endocrinol. Metab. 11, 212-217 (2000)

32. Mosaferi B, Babri S, Ebrahimi H, Mohaddes G: Enduring effects of post-weaning rearing condition on depressive- and anxiety-like behaviors and motor activity in male rats. Physiol. Behav. 142, 131-136 (2015)

33. Naar-King S, Idalski A, Ellis D, Frey M, Templin T, Cunningham PB, Cakan N: Gender differences in adherence and metabolic control in urban youth with poorly controlled type 1 diabetes: the mediating role of mental health symptoms. J. Pediatr. Psychol. 31, 793-802 (2006)

34. Pradhan AD, Manson JE, Rifai N, Buring JE, Ridker PM: C-reactive protein, interleukin 6, and risk of developing type 2 diabetes mellitus. JAMA 286, 327-334 (2001)

35. Pushparaj P, Low H, Manikandan J, Tan B, Tan C: Anti-diabetic effects of Cichorium intybus in streptozotocininduced diabetic rats. J. Ethnopharmacol. 111, 430-434 (2007)

36. Ramanathan M, Jaiswal AK, Bhattacharya SK: Differential effects of diazepam on anxiety in streptozotocin induced diabetic and non-diabetic rats. Psychopharmacology (Berl.) 135, 361-367 (1998) 
37. Reis JS, Amaral CAV, Volpe CMO, Fernandes JS, Borges EA, Isoni CA, Anjos PMFD, Machado JAN: Oxidative stress and interleukin- 6 secretion during the progression of type 1 diabetes. Arq. Bras. Endocrinol. Metab. 56, 441-448 (2012)

38. Roupa Z, Koulouri A, Sotiropoulou P, Makrinika E, Marneras X, Lahana I, Gourni M: Anxiety and depression in patients with type 2 diabetes mellitus, depending on sex and body mass index. Health Sci. J. 3, 32-40 (2009)

39. Shiqing JYYCZ: Study on hypoglycemic effect of Cornus Officinalis [J]. Pharmacol. Clin. Chinese Materia. Medica. 1, 009 (1989)

40. Siddiqui S: Depression in type 2 diabetes mellitus - a brief review. Diabetes Metab. Syndr. 8, 62-65 (2014)

41. Smith KJ, Béland M, Clyde M, Gariépy G, Pagé V, Badawi G, Rabasa-Lhoret R, Schmitz N: Association of diabetes with anxiety: a systematic review and meta-analysis. J. Psychosom. Res. 74, 89-99 (2013)

42. Srinivasan S, Stevens M, Wiley JW: Diabetic peripheral neuropathy: evidence for apoptosis and associated mitochondrial dysfunction. Diabetes 49, 1932-1938 (2000)

43. Stuart MJ, Baune BT: Depression and type 2 diabetes: inflammatory mechanisms of a psychoneuroendocrine comorbidity. Neurosci. Biobehav. Rev. 36, 658-676 (2012)

44. Swaroop JJ, Rajarajeswari D, Naidu J: Association of TNF- $\alpha$ with insulin resistance in type 2 diabetes mellitus. Indian J. Med. Res. 135, 127-130 (2012)

45. Van Tilburg MA, Mccaskill CC, Lane JD, Edwards CL, Bethel A, Feinglos MN, Surwit RS: Depressed mood is a factor in glycemic control in type 1 diabetes. Psychosom. Med. 63, 551-555 (2001)

46. Vinagre I, Sánchez-Quesada JL, Sánchez-Hernández J, Santos D, Ordoñez-Llanos J, De Leiva A, Pérez A: Inflammatory biomarkers in type 2 diabetic patients: effect of glycemic control and impact of LDL subfraction phenotype. Cardiovasc. Diabetol. 13, 34 (2014)

47. Vogelzangs N, Beekman A, De Jonge P, Penninx B: Anxiety disorders and inflammation in a large adult cohort. Transl. Psychiatry 3, e249 (2013)

48. Wang W, Sun F, An Y, Ai H, Zhang L, Huang W, Li L: Morroniside protects human neuroblastoma SH-SY5Y cells against hydrogen peroxide-induced cytotoxicity. Eur. J. Pharmacol. 613, 19-23 (2009)

49. Yamabe N, Noh JS, Park CH, Kang KS, Shibahara N, Tanaka T, Yokozawa T: Evaluation of loganin, iridoid glycoside from Corni Fructus, on hepatic and renal glucolipotoxicity and inflammation in type 2 diabetic $\mathrm{db} / \mathrm{db}$ mice. Eur. J. Pharmacol. 648, 179-187 (2010)

50. Yao R-Q, Zhang L, Wang W, Li L: Cornel iridoid glycoside promotes neurogenesis and angiogenesis and improves neurological function after focal cerebral ischemia in rats. Brain Res. Bull. 79, 69-76 (2009) 